\title{
Sample Rotation: A Mechanical Method to Compensate for Irregular Sample Geometry during Electron Probe Microanalysis of Particles
}

\section{C.S. Schwandt}

\author{
McCrone Associates, Inc., 850 Pasquinelli Drive, Westmont, IL 60559
}

Achieving the most accurate and precise results from electron probe microanalysis (EPMA) requires the analyst to adhere to the tenets of EPMA developed over the past sixty years. Many of the principles were outlined by Castaing [1] and numerous people have improved the technique since that time. EPMA practitioners are well versed, or should be, in these many guidelines that must be followed to produce high quality quantitative analyses. One tenet that is commonly overlooked is the requirement of having flat highly polished standards and samples, and placing them correctly into an electron microprobe with wavelength dispersive spectrometers aligned to the same point on the sample at the point of perpendicular intersection of the electron beam with the sample. Many of us have dealt with the frustrating experience of demonstrating to clients or other practitioners that their poor data are the result of poor sample preparation. While some tenets have been known for sixty years, this one seems to need to be learned repeatedly on a regular basis.

Having just extolled the virtues of flat samples, an about-face is in order. Although the most accurate quantitative analyses and results with the lowest limits of detection require flat samples, there are instances where flat samples are not an option. Particulate samples, or particles on the scale of one to twenty-five micrometers, comprise the cause of many materials failures. Examples of such particles are inclusions in metals, contaminants in liquids, solids, and polymer films. In most cases these tiny pieces of forensic evidence are uniquely characteristic, small, and singular. The analyst has one opportunity to isolate the characteristic particle and analyze it. With a typical size of only ten micrometers in maximum dimension (or less), particles are exceedingly difficult to mount and polish flat, especially without access to dual-column instruments. Extensive sample preparation can be especially risky if only one particle exists. Therefore, analyzing it as it is works best. Although it can be analyzed by energy dispersive X-ray spectrometry, the energy resolution and sensitivity of wavelength dispersive X-ray spectrometry can be worth the extra effort to use. A small decrease in accuracy in terms of absolute concentration calculation is often acceptable in exchange for better energy resolution and having a better understanding of the presence or absence of elements of interest, especially in energy ranges containing many overlaps and when examined with lower resolution EDS systems; for example, energies less than $5 \mathrm{keV}$.

Corrections for particle geometry have been implemented in software like DTSA-II and Probe for EPMA based on the approach and algorithms outlined in Goldstein et al. [2]. The corrections are based on standard morphologies such as the sphere, cube, or prism. Unfortunately most particles do not possess one of these ideal morphologies. Instead, most particles have irregular or combination morphologies. 
To compensate for irregular, non-ideal particle morphology, it is proposed that spinning the particle about a rotational axis parallel to the electron beam during X-ray acquisition presents each WDS spectrometer with total accumulated X-ray intensities that resemble the intensities emanating from a sphere. These intensities can then be treated as if they were generated by physical spheres and the spherical corrections applied to them. Multiple revolutions of the sample are required for acquisition of X-rays at the element peak and each background spectrometer position.

As an initial test case, an irregularly-shaped (subhedral with facture faces) zircon about $20 \mu \mathrm{m}$ long has been mounted on the second hand pin of a mechanical watch using a small amount of soluble gum (Figures 1a, b). The pin is approximately $100 \mu \mathrm{m}$ in diameter. With some trial and error, the particle can be mounted in the rotational center of the pin. The rotation of the second hand is too slow for general common use, requiring excessive acquisition times; however it is sufficient as a concept demonstration. Complete results of extended-time trial collections corrected using the spherical model particle corrections will be presented at the conference.

For special cases where WDS is required and one has the skills required to mount the particle, this is a viable method; however, it requires a mechanical device with a higher rotational rate.

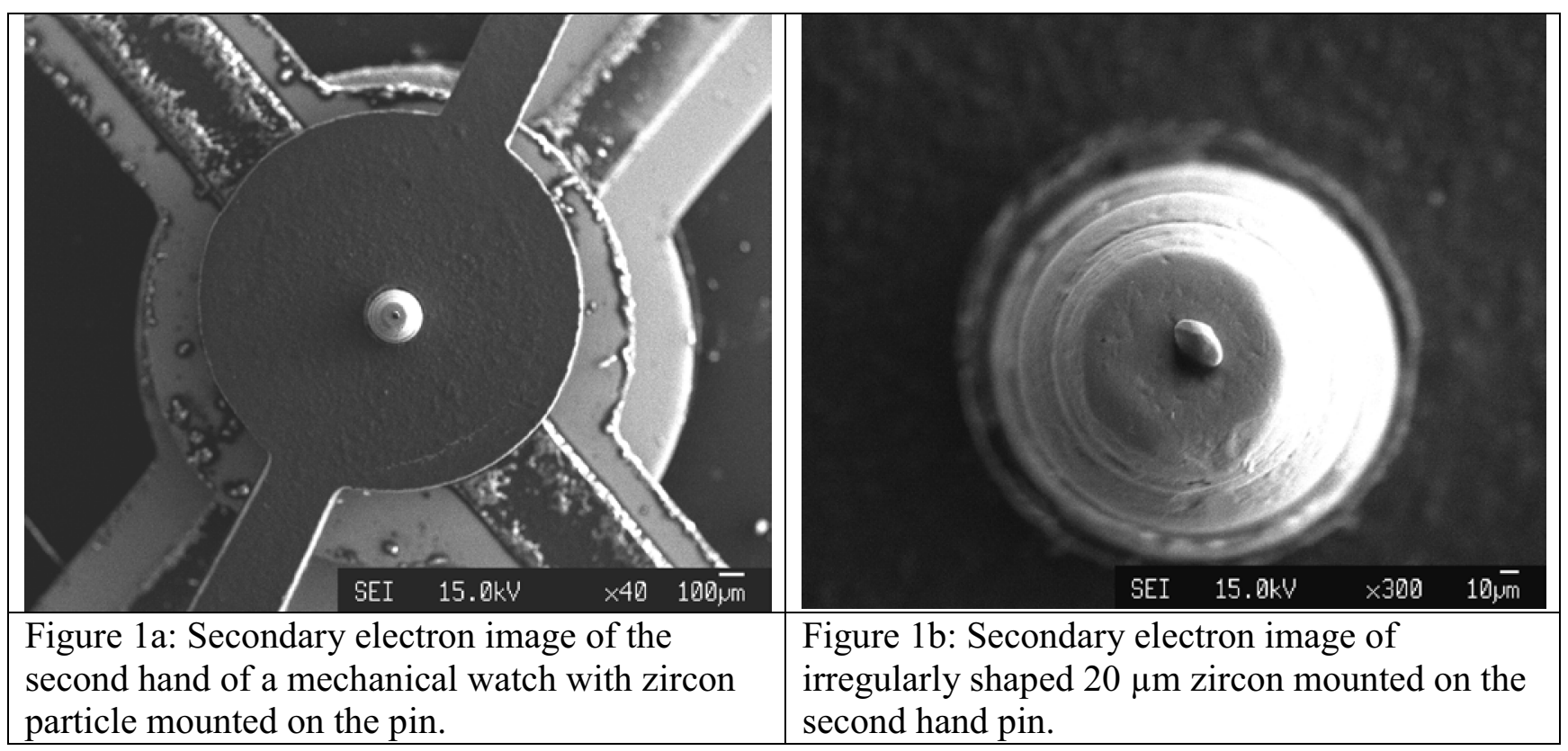

[1] Heinrich, K.F. J., Electron Beam X-ray Microanalysis, Van Nostrand Rheinhold, New York, 1981.

[2] Goldstein, J., Newbury, D., Joy, D., Lyman, C., Echlin, P., Lifshin, E., Sawyer, L., and Michael, J., Scanning Electron Microscopy and X-ray Microanalysis $3^{\text {rd }}$ Ed., Springer, New York, 2003 [3] Thank you to Patrice Anleitner, McCrone Associates, for mounting the particle. Her optical microscopy/sample manipulation skill is extremely appreciated. 